\title{
Neue Strategien zur Integration von Qualität in das Versorgungsmanagement
}

Die medizinische Versorgungsqualität in den Industriestaaten hat ein sehr hohes Niveau erreicht. Dennoch ist unbestritten, dass es nach wie vor Unterschiede in der Ergebnisqualität und damit auch weiteres Verbesserungspotenzial gibt [5]. Dies betrifft einerseits das „Outcome“ bei wichtigen, schweren Krankheitsbildern oder Operationen, bei denen in den letzten Jahren gezeigt werden konnte, dass die Einhaltung von Leitlinien und damit die medizinisch adäquate Prozessorganisation das Ergebnis - beispielsweise in Form der Sterblichkeit - erheblich beeinflussen kann [3]. Aber auch bei Krankheitsbildern und Eingriffen mit niedrigem Risiko lässt sich die Rate der seltenen Todesfälle weiter reduzieren. Bei letzteren ist in diesem Zusammenhang eher von Patientensicherheit die Rede, letztlich ist aber auch diese eine Form der Ergebnisqualität. Es besteht ein weitgehender Konsens, dass die Zahl der vermeidbaren Todesfälle im Krankenhaus in Deutschland insgesamt jährlich zwischen 20000 und 40000 liegen dürfte. Diese Zahl ist ein Vielfaches der 4467 Todesopfer im Straßenverkehr. Die Notwendigkeit für weitere Qualitätsverbesserungen ist daher auch in den entwickelten Ländern gegeben.

Die Methoden zur Messung der Ergebnisqualität konnten in den letzten Jahren erheblich weiterentwickelt werden. International zeichnet sich $\mathrm{ab}$, dass insbesondere im stationären Bereich die medizinischen Informationen, die vor allem im Rahmen der Abrechnungsdaten (DRG) erfasst und gemeldet werden, zunehmend auch für die Qualitätsmessung genutzt werden. In den USA wurden von der „Agency for Healthcare Research and Quality“ (AHRQ) entsprechende Indikatoren entwickelt, in Deutschland haben die Helios Kliniken die Entwicklung eigener, noch detaillierterer Kennzahlen vorangetrieben. Letztere haben Eingang in entsprechende Aktivitäten in der Schweiz und Österreich gefunden [2]. In Deutschland wird die Weiterentwicklung und Nutzung der Ergebnisindikatoren von der Initiative Qualitätsmedizin ( $\left.\mathrm{IQ}^{\mathrm{M}}\right)$ gefördert. Basierend auf den Erfahrungen der Helios Kliniken, die zeigen, dass Managementmethoden einen direkten Einfluss auf die Ergebnisqualität haben können [4], wird diese Initiative vor allem die Integration der Qualitätsmessung und der Qualitätsverbesserung in das Krankenhausmanagement vorantreiben. Hierzu gehört insbesondere die Weiterentwicklung der Peer-Review- Verfahren.
In einem Gemeinschaftsprojekt des AOK Bundesverbandes, des Wissenschaftlichen Instituts der AOK (WIdO), des Forschungs- und Entwicklungsinstituts für das Sozial- und Gesundheitswesen Sachsen-Anhalt (FEISA) und der Helios Kliniken wurden in Deutschland ferner erstmals sektor-übergreifende Methoden zur Messung von Langzeitergebnissen aus den Daten der Krankenkassen entwickelt [1]. Ein QSR-Klinikbericht steht zur Verfügung, der interessierte Kliniken über Langzeitergebnisse ihrer Behandlung informiert. Durch das WIdO wurde unlängst die Entwicklung von Qualitätsindizes vorangetrieben um Ergebnisqualität breiter zu erfassen und auch bei Kliniken mit kleinen Fallzahlen sicher und reliabel beurteilen zu können. Die AOK arbeitet an verschiedenen Stellen an der Nutzbarmachung von QSR-Informationen zur Ergebnisqualität sowohl für Versicherten- und Patienteninformation als auch im Kontext von Versorgungsverträgen.

Die verschiedenen neuen Methoden sind geeignet, um bestehende, bewährte Ansätze zur Sicherung der Strukturqualität zu ergänzen und zu erweitern. Einige Verfahren zur Messung von Prozess-Schritten können sie möglicherweise sogar im Sinne einer weiter gefassten Ergebnisorientierung ersetzen. Hinsichtlich der Strukturqualität sei beispielsweise auf die Vereinbarungen nach §135(2) SGB V verwiesen. Diese stellen gerade bei neuen Verfahren die Mindestqualität sicher und sind ferner im Sinne eines „Ja/Nein“Ansatzes (erfüllt/nicht erfüllt) auch jetzt schon vergütungsrelevant. „Pay for Performance (P4P)“ findet also in diesem Sinne hier, wie auch im $\mathrm{Zu}$ sammenhang mit manchen anderen Vorgaben im Gesundheitswesen, bereits statt, auch wenn dies oft nicht unter diesem Aspekt wahrgenommen wird.

Vor dem Hintergrund der neueren Möglichkeiten stellt sich die Frage, wie sich die nationalen Qualitätsstrategien künftig entwickeln werden. Unsere Tagung wird neben aktuellen Berichten aus den USA vor allem die Ansätze aus der Schweiz, Österreich und Deutschland vorstellen. Für Deutschland wird natürlich insbesondere die weitere Entwicklung nach den Entscheidungen des Gemeinsamen Bundesausschusses zur Debatte stehen.

Die Entwicklung von geeigneten Methoden für „Pay for Performance“ wird international als eines der wichtigen Themen bei der Weiterentwicklung der Vergütungssysteme gesehen [7]. Bis
T. Mansky ${ }^{1}$
M. Heberer ${ }^{2}$
J. Klauber $^{3}$

Qualitätsmanagement

Qualitatsmanagement

Institut

1 HELIOS Kliniken

2Universitätsspital Basel

${ }^{3}$ Wissenschaftliches Institut der AOK

Bibliografie

DOI 10.1055/s-0029-1242686 Dtsch Med Wochenschr 2009; 134: S301-S302 - @ Georg Thieme Verlag KG Stuttgart . New York · ISSN 0012-0472

Korrespondenz PD Dr. Thomas Mansky Leiter Medizinische Entwicklung HELIOS Kliniken

Friedrichstr. 136 10117 Berlin Tel. 030/521 321-140 eMail thomas.mansky@ helios-kliniken.de 
jetzt wirken sich aber Ergebnismessungen in Deutschland und den Nachbarländern weder auf die Krankenhausvergütung noch auf die Versorgungssteuerung aus, auch wenn sie mittelbar durchaus Patienten- und Zuweiserentscheidungen beeinflussen können. Sie werden daher in den Fachzeitschriften und vielfach auch bei den Leistungserbringern oft nur der Rubrik „Marketingaktivitäten“ zugeordnet. Dies ist angesichts der eingangs dargestellten medizinischen Bedeutung des Themas unzureichend. Es stellt sich daher die Frage, ob, wann und in welcher Form Ergebniskennzahlen Eingang in die Vergütung finden könnten. Dazu sollen am zweiten Tag Erfahrungen der Krankenkassen mit bisherigen Projekten, beispielsweise im Rahmen von Verträgen der integrierten Versorgung, mögliche künftige Konzepte der Kassen, der Bundesärztekammer und wichtiger anderer Beteiligter sowie auch einige ausgewählte internationale Projekte vorgestellt werden.

Neben den institutionellen Verfahren ist die Vermittlung wichtiger medizinischer Ergebniskennzahlen an Versicherte bzw. Patienten und Angehörige ein eigenstän- diger Problembereich. Angesichts einer immer größeren „Informationsvernebelung“ in den gesetzlichen Qualitätsberichten, die jüngst im „Petrophagenextraktor" eine würdige, selbst-satirische Kommentierung fand [6], stellen wir in einem Vortragsblock die Frage in den Mittelpunkt, ob und wie es gelingen kann, Patienten und Versicherte über fachlich wesentliche medizinische Ergebnisse angemessen zu informieren.

Wir hoffen, auch in diesem Jahr mit einem auf aktuelle strategische Fragestellungen ausgerichteten Exzerpt aus den vielfältigen Aktivitäten im Bereich des Qualitätsmanagements wieder ein Forum für Innovation, Information und Meinungsbildung an der Schnittstelle von medizinischer Qualität, Krankenhausmangement und Vergütungssystematik bieten zu können.
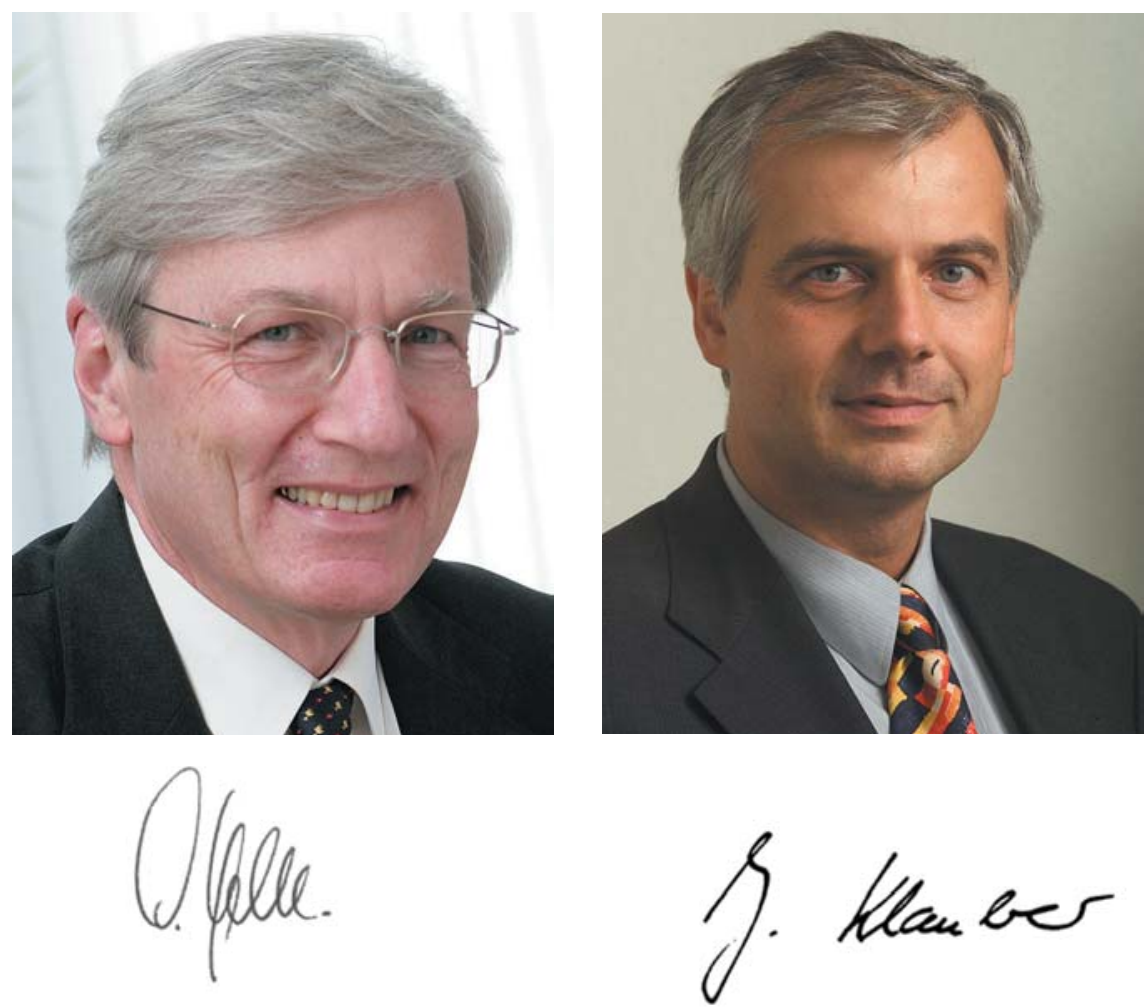

Prof. Dr. Michael Heberer

Universitätsspital Basel

Literatur

1 AOK-Bundesverband, Forschungs- und Entwicklungsinstitut für das Sozial- und Gesundheitswesen Sachsen-Anhalt (FEISA), HELIOS Kliniken, Wissenschaftliches Institut der AOK (WIdO). Qualitätssicherung der stationären Versorgung mit Routinedaten (QSR) - Abschlussbericht. Bonn: WIdO, 2007

2 Bundesamt für Gesundheit BAG. Qualitätsindikatoren der Schweizer Akutspitäler 2006. Bern: Bundesamt für Gesundheit, 2009

3 Peterson ED et al. Association Between Hospital Process Performance and Outcomes Among Patients With Acute Coronary Syndromes. JAMA 2006; 295: 1912-1920

4 HELIOS Kliniken Gruppe. Ergebnisqualität sicher messen und aktiv verbessern - Erfahrungen. Medizinischer Jahresbericht 2006/2007. Berlin: HELIOS Kliniken, 2008

5 Kohn LT, Corigan JM, Donaldson MS. (Ed.) To err is human - Building a safer health system. Washington D.C.: Institute of medicine, 1999

6 Krause T. Randnotiz. Deutsches Ärzteblatt 2009; 106 (38): A1815

7 Porter ME, Olmsted Teisberg E. Redefining Health Care. Creating Value-Based Competition on Results. Boston: Harvard Business School Publishing, 2006

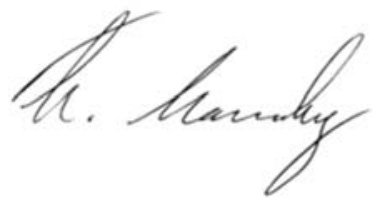

PD Dr. Thomas Mansky HELIOS Kliniken 